\title{
Nutzung elektronischer Zigaretten (E-Zigaretten) und E-Shishas durch Kinder und Jugendliche
}

\author{
Evidenzpapier der Gemeinsamen Suchtkommission \\ der kinder-und jugendpsychiatrischen und \\ psychotherapeutischen Fachgesellschaft und \\ Verbände (DGKJP, BAG, BKJPP)
}

Nicolas Arnaud ${ }^{1}$, Martin Holtmann², Peter Melchers ${ }^{3}$, Marianne Klein 4 , Gisela Schimansky ${ }^{5}$, Thomas Krömer ${ }^{6}$, Olaf Reis ${ }^{7}$ und Rainer Thomasius ${ }^{1}$

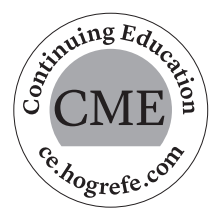

\author{
Deutsches Zentrum für Suchtfragen des Kindes- und Jugendalters (DZSKJ), Universitätsklinikum Hamburg-Eppendorf, Hamburg \\ LWL-Universitätsklinik Hamm der Ruhr-Universität Bochum, Klinik für Kinder- und Jugendpsychiatrie, Psychotherapie und \\ Psychosomatik, Hamm \\ Klinik für Kinder- und Jugend psychiatrie, Psychosomatik und Psychotherapie, Klinikum Oberberg, Kreiskrankenhaus Gummersbach \\ und Klinik Marienheide, Gummersbach \\ Klinikum Schloss Winnenden, Winnenden \\ Praxis für Kinder- und Jugend psychiatrie und Psychotherapie Dr. Schimansky, Hannover \\ Gemeinschaftspraxis für Kinder- und Jugendpsychiatrie, Psychotherapie \& Psychosomatik, Hamburg \\ Klinik für Psychiatrie, Neurologie, Psychosomatik und Psychotherapie im Kindes- und Jugendalter, Universitätsmedizin Rostock, Rostock
}

\begin{abstract}
Zusammenfassung: Die besonderen Risiken des Konsums von elektronischen Zigaretten (E-Zigaretten) im Kindes- und Jugendalter werden im gesundheitspolitischen Diskurs zu wenig berücksichtigt. Im vorliegenden Beitrag werden die aktuellen Verbreitungs- und Konsummuster von E-Zigaretten sowie das Gefährdungspotenzial einer Nutzung für Kinder und Jugendliche bewertet. Auf Basis aktueller nationaler und internationaler Studien zeigt sich seit einigen Jahren eine deutliche Zunahme des Konsums von E-Zigaretten, die im scharfen Kontrast zum rückläufigen Tabakkonsum bei Kindern und Jugendlichen in Deutschland steht. Dabei konsumieren junge Menschen ohne Tabakerfahrung häufiger E-Zigaretten als Gleichaltrige mit einem gelegentlichen und regelmäßigen Tabakgebrauch. Sie experimentieren außerdem häufiger mit konventionellen Zigaretten, wenn sie zuvor E-Zigaretten konsumiert haben. Die weitgehend unregulierte Verfügbarkeit von E-Zigarettenprodukten der neuesten Generation wie JUUL hat unter Schüler_innen in den USA zu einer dramatischen Zunahme minderjähriger E-Zigarettenkonsument_ innen geführt. Diese Produkte werden mit hohem Nikotingehalt und aromatischen Geschmacksrichtungen angeboten und über Werbung und soziale Medien intensiv als trendige Lifestyleprodukte für junge Nutzergruppen vermarktet. Auch in Deutschland nehmen diese Produkte stark an Relevanz zu. Die Erfolge der Tabakprävention der letzten Jahre werden durch wirksame Werbung für E-Zigaretten gefährdet. Die Suchtkommission der deutschen kinder- und jugendpsychiatrischen Verbände und wissenschaftlichen Fachgesellschaft spricht sich daher für ein umgehendes, striktes und umfassendes Werbeverbot für E-Zigaretten aus.
\end{abstract}

Schlüsselwörter: Elektronische Zigaretten, Tabakkonsum, Nikotin, Kinder und Jugendliche, Suchtstörungen 
Use of Electronic Cigarettes (e-cigs) and e-Shishas by Children and Adolescents: Evidence Paper of the Joint Addiction Commission of the German Societies and Professional Associations of Child and Adolescent Psychiatry and Psychotherapy

\begin{abstract}
The particular risks associated with the consumption of electronic cigarettes (e-cigs) in children and adolescents are not sufficiently considered in the health policy discourse. The present article evaluates the current dissemination and consumption patterns of e-cigarettes as well as the health risks attached to children and adolescents. Based on data from current national and international studies, there has been a clear increase in the consumption of e-cigarettes over the past years. This stands in sharp contrast to the overall decline in tobacco consumption among both children and adolescents in Germany. Young people without tobacco experience are now consuming more frequently e-cigarettes than those who occasionally or regularly use tobacco. They also are experimenting more frequently with conventional cigarettes if they have previously consumed e-cigarettes. The largely unregulated availability of e-cigarette products to the newest generation, such as JUUL, led to a dramatic increase in their prevalence among high-school students in the USA. Products with high nicotine content and multiple flavors are being marketed intensively as trendy lifestyle products to young user groups via advertising and social media campaigns. These products are also becoming increasingly relevant in Germany. The success of tobacco prevention in recent years is presently jeopardized by the ongoing effective advertising for e-cigarettes. The Addiction Commission of the German Child and Youth Psychiatric Federations and Scientific Societies therefore call for an immediate, strict, and comprehensive ban of e-cigarette advertising.
\end{abstract}

Keywords: e-cigarettes, tobacco use, nicotine, children and adolescents, addictive disorders

\section{Einleitung}

Elektronische Zigaretten (E-Zigaretten), E-Shisha und Tabakerhitzer gehören zu den Electronic (Non-)Nicotine Delivery Systems (ENDS), die alternativ oder ergänzend zum Rauchen von herkömmlichen Tabakprodukten verwendet werden. Im Gegensatz zur Tabakzigarette wird in der E-Zigarette kein Tabak verbrannt und Konsumenten inhalieren keinen Rauch verbrennender Pflanzenteile. E-Zigaretten sind batteriebetriebene Inhalationsgeräte, die eine Flüssigkeit („Liquid“, hauptsächlich bestehend aus Propylenglycol, Glycerin, Wasser und Lebensmittelaromen) zu einem Aerosol vernebeln (Kotz et al., 2018). Sie kamen vor gut zehn Jahren ohne fundiertes Wissen über die gesundheitlichen Risikopotenziale (etwa hinsichtlich der kanzerogenen Aerosol-Bestandteile und dem Suchtpotenzial von nikotinhaltigen Liquiden) in Deutschland auf den Markt. Liquids sind mit und ohne Nikotin (in unterschiedlicher Konzentration) sowie in den verschiedensten Geschmacksrichtungen erhältlich. Vertrieben werden verschiedene Arten von E-Zigaretten, die sich etwa in der Leistung und der Art der Nachfüllung unterscheiden. Unter Jugendlichen sind sogenannte E-Shishas beliebt (Orth \& Merkel, 2020), die in der Regel kein Nikotin enthalten. Die Einwegprodukte gleichen im Aufbau und Äußeren der E-Zigarette, sind bunt bedruckt und haben ein Mundstück vergleichbar einer Wasserpfeife. Seit Juni 2016 sind in Deutschland zudem Tabakerhitzer auf dem Markt, eine Variante der E-Zigarette, die seit der Markteinführung massiv in der Öffentlichkeit beworben wird, bei jungen Menschen aber vergleichsweise weniger verbreitet scheint (Orth \& Merkel, 2020). Im Gegensatz zur E-Zigarette kommt bei diesen Geräten echter Tabak zum Einsatz, der auf etwa $350^{\circ} \mathrm{C}$ erhitzt, aber nicht verbrannt wird (weshalb diese Geräte im Englischen auch „Heat-not-burn“-Produkte genannt werden). Da echter Tabak verwendet wird, enthält das Aerosol von Tabakerhitzern grundsätzlich Nikotin, und zwar über $80 \%$ der Menge herkömmlicher Tabakzigaretten ( $84 \%$ im Fall des untersuchten Produktes (IQOS) bei einer Nikotinmenge von $301 \mu \mathrm{im}$ Vergleich zu $361 \mu \mathrm{g}$; Auer et al., 2017). Der (noch seltene) Konsum anderer psychoaktiver Substanzen als Nikotin mit Hilfe der E-Zigarette, wie zum Beispiel Cannabinoiden, Amphetaminen etc. wird hier nur am Rande thematisiert.

Die möglichen Risiken des Gebrauchs ${ }^{1}$ von E-Zigaretten haben in den letzten Jahren auch unter Medizinern und Gesundheitswissenschaftlern eine lebhafte Diskussion ausgelöst. Mehrere nationale und internationale Institutionen, Expertengruppen und Fachgesellschaften haben in den letzten fünf Jahren Empfehlungen für den Umgang mit EZigaretten veröffentlicht. National haben beispielsweise das Deutsche Krebsforschungszentrum (DKFZ, 2015), die Deutsche Hauptstelle für Suchtfragen e.V. (DHS, 2016), die Deutsche Gesellschaft für Pneumologie und Beatmungsmedizin e.V. (2020; Nowak et al., 2015) sowie der Dachverband der Suchtfachgesellschaften (Rüther et al., 2017) und international das Britische National Center for Smoking Cessation and Training (2016) sowie die US-amerikanische National Academy of Science (Eaton et al., 2018) entsprechende Stellungnahmen herausgegeben. Im Allgemeinen beziehen sich diese Einschätzungen und Empfehlungen auf den E-Zigarettengebrauch von Erwachsenen. Oftmals steht in den vorhandenen Stellungnahmen eine Abwägung der gesundheitlichen Risiken und der Chancen der E-Zigarette

Gesundheitspolitisch diskutiert werden neben den Risiken auch mögliche Chancen von E-Zigaretten diskutiert und hierbei insbesondere die Frage ob sich E-Zigaretten zum Einsatz in der Tabak- und/oder Nikotinentwöhnung bei Rauchern eignen. Diese Maßnahme zur Schadensbegrenzung spielt im Kindes- und Jugendalter eine untergeordnete Rolle und wird in dieser Stellungnahme daher nur am Rande behandelt. 
als Ausstiegshilfe aus dem gewohnheitsmäßigen Tabakkonsum im Vordergrund (vgl. hierzu die Ergebnisse einer aktuellen Cochrane-Übersichtsarbeit Hartman-Boyce et al., 2020). Die besonderen Bedingungen und Auswirkungen des Konsums im Kindes- und Jugendalter finden eher am Rande und in Form von Empfehlungen zum Jugendschutz Berücksichtigung. Im vorliegenden Beitrag (eine Kurzfassung ist bereits als Stellungnahme publiziert worden: Deutsche Gesellschaft für Kinder- und Jugendpsychiatrie, Psychosomatik und Psychotherapie e.V. [DGKJP], Bundesarbeitsgemeinschaft der Leitenden Klinikärzte für Kinderund Jugendpsychiatrie, Psychosomatik und Psychotherapie e.V. [BAG KJPP], Berufsverband für Kinder- und Jugendpsychiatrie, Psychosomatik und Psychotherapie in Deutschland e.V. (BKJPP), 2020) werden die Verbreitungs- und Konsummuster sowie das Gefährdungspotenzial einer Nutzung von E-Zigaretten für Kinder und Jugendliche bewertet. Sie strukturiert sich im Wesentlichen anhand der folgenden vier Fragestellungen:

1. Wie verbreitet ist der E-Zigarettenkonsum im Kindesund Jugendalter?

2. Welche kurz- und langfristigen Risiken des Konsums der E-Zigarette sind bekannt?

3. Trägt der zunehmende Gebrauch von E-Zigaretten im öffentlichen Raum zu einer Re-normalisierung des Rauchens bei und konterkariert womöglich bisherige Erfolge in der Tabakprävention?

4. Bahnt die Nutzung der E-Zigarette bei Jugendlichen den Übergang zum Tabakrauchen an?

\section{Wie verbreitet ist der E-Zigarettenkonsum im Kindes- und Jugendalter?}

Unter den 12- bis 25-jährigen Kindern, Jugendlichen und jungen Erwachsenen in Deutschland entwickelt sich das konventionelle Tabakrauchen seit Jahren stark rückläufig und ist derzeit auf einem historischen Tiefstand (Orth \& Merkel, 2020). Seit einigen Jahren ist in dieser Altersgruppe allerdings ein Anstieg des Konsums von E-Zigaretten zu verzeichnen (Orth \& Merkel, 2018; 2020). Die aktuelle Lebenszeitprävalenz, die auf einen mindestens experimentellen Konsum von E-Zigaretten hinweist, liegt repräsentativen Daten der Bundeszentrale für gesundheitliche Aufklärung (BZgA; Orth \& Merkel, 2020) zufolge bei Jugendlichen bei $14,5 \%$ und damit zumindest in dieser Altersgruppe (12 bis 17 Jahre) erstmals über der Lebenszeitprävalenz des Tabakkonsums (14\%). Die 30-Tagesprävalenz, die auf einen aktuellen Gebrauch hinweist, ist mit 4,1\% deutlich niedriger und liegt auch noch etwas unterhalb derjenigen des berichteten Tabakgebrauchs $(6,6 \%)^{2}$. Bei den 18 - bis 25 -Jährigen liegt die Lebenszeitprävalenz des E-Zigarettenkonsums mit 32,5\% deutlich höher, aber noch weit unterhalb des Konsums von Tabakzigaretten (53,6\%). Die 30-Tagesprävalenz des E-Zigarettengebrauchs liegt im Vergleich zu den Jugendlichen auf einem nur geringfügig höheren Niveau von $6,9 \%$ und identifiziert den Tabakkonsum $(27,8 \%)^{3}$ bei den jungen Erwachsenen als das deutlich verbreitetere Verhalten.

Weitere repräsentative Befragungen aus der Allgemeinbevölkerung der letzten Jahre kommen nur zu teilweise vergleichbaren Ergebnissen. Bei Eichler und Kollegen (2016) berichteten die 330 Befragten in der Altersgruppe der 14- bis 19-Jährigen eine Lebenszeitprävalenz des E-Zigarettenkonsums von 20,3\%, wobei ein Großteil (13,9\%) angab, E-Zigaretten lediglich probiert zu haben. Der Anteil regelmäßiger Konsumenten von E-Zigaretten unter den Adoleszenten betrug nur 0,9\% und lag damit deutlich niedriger als in der Gesamtbevölkerung $(1,4 \%)$.

In der etwas aktuelleren „Deutschen Befragung zum Rauchverhalten" (DEBRA-Studie, Kotz et al., 2018; Kotz \& Kastaun, 2018) lag der Anteil ,aktueller E-Zigarettennutzer" unter den 14- bis 17-Jährigen bei 2,9 \% (als ,,aktuelle Tabak-Zigarettennutzer" bezeichneten sich hingegen 11,9\%). Schneider und Kollegen (2017) kommen auf Basis einer Auswertung von Daten aus dem nationalen Krebsmonitoring auf einen Anteil von $20 \%$ aktueller Konsumenten unter den 14- bis 17-Jährigen mit E-Zigarettenerfahrung.

Aussagen zu signifikanten Trendentwicklungen des aktuellen und regelmäßigen Konsums von E-Zigaretten liegen für die deutsche Bevölkerung derzeit nicht vor. Die vorhandenen querschnittlichen Daten zeigen aber deskriptiv einen Anstieg: Der Anteil derjenigen, die im Rahmen der Drogenaffinitätsstudie der BZgA angeben, in den letzten 30 Tagen E-Zigaretten konsumiert zu haben, erhöhte sich - bei identischer Befragungsmethodik - unter den 12- bis 17-Jährigen seit dem Jahr 2012 von 2,6\% auf $4,1 \%$ in 2019 (Orth \& Merkel, 2020). Der Anteil der aktuellen E-Zigarettennutzer_innen ist insgesamt von 2016 bis 2017 in der Allgemeinbevölkerung (14 Jahre und älter) deskriptiv von 1,2\% auf etwa 2,0\% angestiegen (Kotz et al., 2018). Aus anderen Ländern in Europa, etwa dem Vereinigten Königreich werden vergleichbar hohe Zuwachsraten berichtet (Ernst \& Young, 2017).

Im internationalen Vergleich liegen die Prävalenzen des E-Zigarettenkonsums unter Jugendlichen in Deutschland etwa im Mittelfeld. Aktuelle Daten aus der ESPAD-Studie (European School Survey Project on Alcohol and Other

davon berichten 2,9\% der Befragten einen ausschließlichen und 3,7\% einen kombinierten Konsum weiterer nicht brennbarer Nikotinprodukte. 16,4\% ausschließlich Tabakzigarette; 11,1\% kombinierter Konsum; 1,2\% konsumieren Wasserpfeife und E-Produkte bzw. Tabakerhitzer aber keine Tabakzigarette. 
Drugs; ESPAD Group, 2020) mit Daten von über 100000 Schüler_innen im Alter von 15 bis 16 Jahren aus 35 Ländern in Europa zeigen für deutsche Teilnehmende eine berichtete 30 -Tagesprävalenz von $16 \%$ (ESPAD-Mittelwert: $14 \%$ ), 12-Monatsprävalenz von 33\%) ESPAD-Mittelwert: $28 \%$ ), und Lebenszeitprävalenz von 42\% (ESPAD-Mittelwert: $40 \%$ ). Eine vergleichende Untersuchung (Yoong et al., 2018) zur globalen Verbreitung in 13 industrialisierten Ländern (aus Europa, Asien, Nordamerika und Neuseeland; Deutschland war nicht darunter) zeigte sehr heterogene Prävalenzen und Zuwachsraten im E-Zigarettenkonsum zwischen den Jahren 2008 und 2015. Die Lebenszeitprävalenzen lagen zwischen 5,9\% (Italien) und 62,1\% (Polen).

Dass sich die Prävalenzraten des E-Zigarettenkonsums unter Jugendlichen womöglich stark abhängig von der Verfügbarkeit und gesetzgeberischen Regulation entwickeln, legen jüngste Daten (Cullen et al., 2019) aus den vergleichsweise wenig reglementierten USA nahe: Dort beträgt der Anteil der E-Zigarettenkonsument innen an Middle- und Highschools mittlerweile 24,3\% (Wang et al, 2020) und liegt damit um ein Vielfaches über dem etwa durch Werbeverbote vergleichsweise stark reglementierten Konsum konventioneller Tabakzigaretten (10,5\%). Über ein Drittel der Schüler_innen (40\%) berichtet dabei einen gewohnheitsmäßigen bzw. abhängigen Konsum von E-Zigaretten an 20 oder mehr Tagen im Monat (ebd.). Der Großteil der jugendlichen Konsument_innen in den USA greift insbesondere auf E-Zigarettenprodukte mit hohem Nikotingehalt, wie JUUL und Suorin DROP, zurück (ebd.). Diese HightechGeräte der neuesten Generation werden über USB-Ports aufgeladen und verwenden Nikotinpatronen im „pod“-Produktdesign, welche durch sehr ansprechende Werbung und Social-Media-Kampagnen, die sich speziell an Jugendliche $\mathrm{zu}$ richten scheinen, vermarktet werden. Sie werden in verschiedenen Geschmacksrichtungen (vor allem Minz-/Menthol-Geschmacksstoffe sowie Frucht- und Süßwarenaromen) konsumiert und liefern eine Nikotinkonzentration, die mit der einer herkömmlichen Zigarette vergleichbar ist. Auch in Deutschland, wo der Anteil regelmäßiger E-Zigarettenkonsument_innen im Vergleich zu den USA (noch) deutlich niedriger liegt, nehmen diese Produkte an Relevanz stark zu (Schneider et al., 2017).

\section{Welche kurz- und langfristigen Risiken birgt der Konsum der E-Zigarette?}

Zunächst ist anzumerken, dass E-Zigaretten ohne umfangreiche präklinische Studien, toxikologische Untersuchungen oder Sicherheitstests auf den internationalen Märkten zugelassen worden sind (Pisinger \& Døssing, 2014). Eine gesundheitliche Unbedenklichkeit kann aber nicht vorausgesetzt werden. Insbesondere die Frage nach den langfristigen mit dem Gebrauch assoziierten gesundheitlichen Risiken ist nicht zuletzt durch die großen Unterschiede in den verfügbaren E-Zigaretten und den raschen Veränderungen der vermarkteten Produkte (Nicol et al., 2020) sehr umstritten und eine abschließende Klärung schwierig (Reimer et al., 2016).

Die derzeitige Studienlage deutet darauf hin, dass die Freisetzung von Schadstoffen während des Gebrauchs von E-Zigaretten mit gesundheitlichen Risiken verbunden ist, aber im Vergleich zu Tabakzigaretten geringer ausfällt (DKFZ, 2020). Da keine Verbrennung von Stoffen stattfindet, entstehen im Gegensatz zur Tabakzigarette kein Kohlenmonoxid, keine Blausäure, kein Arsen und auch keine krebserzeugenden polyzyklischen aromatischen Kohlenwasserstoffe. Das Risiko für kardiovaskuläre Erkrankungen regelmäßig rauchender Erwachsener kann Studien zufolge bei einem Wechsel von Tabak- zu E-Zigaretten kurzfristig und im 1-Monatsintervall messbar gesenkt werden (George et al., 2019). Allerdings ist davon auszugehen, dass die gesundheitlichen Verbesserungen vermutlich auf diejenigen Raucher innen begrenzt sind, die vollständig auf E-Zigaretten umsteigen und mit dem Rauchen herkömmlicher Tabakzigaretten komplett aufhören (Pisinger \& Døssing, 2014). Der vollständige Umstieg gelingt jedoch selten, E-Zigaretten werden in Deutschland überwiegend zusätzlich zu Tabakzigaretten konsumiert (Kotz \& Kastaun, 2018). Als Ausstiegshilfe aus dem Tabakrauchen können E-Zigaretten einem aktuellen Cochrane Review (Hartmann-Boyce et al., 2020) zufolge aber zumindest kurzfristig effektiver sein als konventionelle Methoden wie etwa eine Nikotin-Ersatztherapie oder verhaltensbezogene Unterstützungsangebote.

Dass der derzeitige Stand des Wissens die gesundheitlichen Risiken des E-Zigarettenkonsums niedriger ausweist als die des Konsums herkömmlicher Zigaretten bedeutet keineswegs, dass der Konsum von E-Zigaretten risikofrei ist. In chemisch-toxikologischen Analysen einzelner Liquide wurden Spuren typischer Tabakgiftstoffe und Kanzerogene (Nitrosamine und schädliche tabakspezifische Alkaloide wie Anabasin, Myosmin und $\beta$-Nicotyrin) nachgewiesen, vermutlich infolge der Gewinnung des Nikotins aus Tabak (Produkte der neuesten Generation wie etwa JUUL verwenden organische Nikotinsalze, die aus Tabakblättern extrahiert werden). Darüber hinaus wurden im Aerosol verschiedener E-Zigaretten Schadstoffe wie Formaldehyd, Acetaldehyd, Acrolein, Nickel und Chrom nachgewiesen (Mishra et al., 2017; Lisko et al., 2015). Studien weisen darauf hin, dass der Konsum von E-Zigaretten erhebliche Auswirkungen auf die Gesundheit der Atemwege hat (Gotts et al., 2019).

Die kurzfristigen Nebenwirkungen der Nutzung von EZigaretten sind ausführlich beschrieben: Reizungen im Mund- und Rachenraum, Husten, Augenreizungen, Kopf- 
schmerz, Schwindel, Übelkeit, Müdigkeit, Schlaflosigkeit. Bei unsachgemäßer Anwendung ist das Eintreten des nikotinhaltigen Liquids in den Mundraum möglich, was gerade für jüngere Kinder fatale Folgen haben kann (Eaton et al., 2018). In Tierversuchen führte die Inhalation von Glycerin und Propylenglycol zu Zellveränderungen im Kehlkopf sowie Reizungen der Nasenschleifhäute bzw. Nasenbluten (Pisinger \& Døssing, 2014). In Tierstudien konnte zudem nachgewiesen werden, dass die pränatale Exposition mit dem Aerosol aus E-Zigaretten zu fetalen Beeinträchtigungen führt (ebd.). Bisher kaum erforscht sind die langfristigen gesundheitlichen Auswirkungen einer wiederholten Inhalation von Propylenglycol etwa auf Atemwegs- und Herzkreislauferkrankungen sowie die Entwicklung von Krebserkrankungen und weitere Entwicklungsrisiken (DKFZ, 2020). Gotts und Kollegen (2019) kommen in ihrem systematischen Literaturüberblick zu dem Schluss, dass gesicherte Aussagen zu den langfristigen Auswirkungen von E-Zigaretten oder eine abschließende Vergleichbarkeit zu den Risiken des Konsums brennbarer Zigaretten nicht möglich sind. Eine im letzten Jahr vorgelegte und in den öffentlichen Medien stark rezipierte US-amerikanische Studie (Bhatta \& Glantz, 2019), die ein um den Faktor 2,25 erhöhtes Herzinfarktrisiko bei täglichen Nutzer_innen von E-Zigaretten ermittelt hatte, wurde wegen fehlerhafter Analysen inzwischen zurückgezogen. Der Einfluss von E-Zigaretten auf das Herzinfarktrisiko ist nach wie vor ungeklärt.

Das Abhängigkeitspotenzial von nikotinhaltigen E-Zigaretten ist unstrittig und bei vergleichbarem Nikotingehalt auch mit dem des herkömmlichen Tabakrauchens vergleichbar (Pisinger \& Døssing, 2014). Die Nikotinexposition durch einen verbreiteten Gebrauch nikotinhaltiger E-Zigaretten wird auch mit einem erhöhten Risiko für die Entwicklung von Suchterkrankungen hinsichtlich weiterer psychotroper und potenziell abhängiger Substanzen in Verbindung gebracht (Kandel \& Kandel, 2014).

Die Neufassung der International Classification of Disease (ICD-11) unterscheidet bei den nikotinbezogenen Suchtstörungen entsprechend nicht mehr zwischen herkömmlichen Zigaretten oder nikotinhaltigen E-Zigaretten (Arnaud \& Thomasius, 2020a). Das Ausmaß des abhängigen Gebrauchs von E-Zigaretten ist unseres Wissens in großangelegten Studien bislang nicht untersucht worden. Einzelne Studien fanden jedoch vergleichbare und teilweise sogar höhere Werte für Nikotinabhängigkeit (gemessen mit dem Fagerström Test for Nicotine Dependence) bei erwachsenen Konsument innen von E-Zigaretten im Vergleich zu Konsument_innen herkömmlicher Zigaretten (Johnson et al., 2018; Jankowski et al., 2019). Derlei vergleichende Studien zu Jugendlichen liegen unseres Wissens keine vor. Ehemalige Tabakraucher_innen, die auf den alternativen Konsum von E-Zigaretten umgestiegen sind, verbleiben jedoch in hohem Maße in einer Nikotinabhängigkeit (Reimer et al., 2016), was der Behauptung eines vergleichsweise geringeren Abhängigkeitspotenzials der E-Zigarette entgegensteht.

Studienvergleiche sind limitiert, da häufig unklar ist, welche Mengen Nikotin bei der Nutzung von E-Zigaretten tatsächlich aufgenommen werden. Selbst Kartuschen mit identischen Inhaltsangaben können je nach Gerät, Füllmenge, Batteriestärke und Intensität, mit der am Gerät gezogen wird, unterschiedliche Mengen Nikotin abgeben. Erhebliche Unterschiede im Hardware-Design und in den Inhaltsstoffen erschweren derzeit allgemeine Aussagen über die potenziellen Schäden von E-Zigaretten. Zudem ist über kombinierte schädliche Effekte („Cocktail Effekt”) des Konsums von E-Zigaretten und herkömmlichen Zigaretten bislang sehr wenig bekannt. Gesundheitlich negative Auswirkungen von Nikotin auf die Gefäßgesundheit sind insbesondere bei Vorliegen einer kardiovaskulären Vorerkrankung zu erwarten (Benowitz \& Burbank, 2016).

Der Konsum anderer psychoaktiver Substanzen als Nikotin mit Hilfe der E-Zigarette wie zum Beispiel Cannabinoiden oder Amphetaminen ist selten, wobei systematische Daten fehlen. Allerdings kam es Ende des Jahres 2019 zu einer Welle von schweren Lungenerkrankungen (EVALI: „e-cigarette, or vaping, product use associated lung injury") mit Todesfolge im Anschluss an den Konsum von E-Zigaretten vor allem bei jugendlichen Konsumenten in den USA. Toxikologische Untersuchungen legen nahe, dass unerlaubte und vermutlich mit Vitamin E-Acetat gestreckte Tetrahydrocannabinol (THC)-haltige Liquids für die schweren Lungenschäden verantwortlich sind (Blount et al., 2020). Entsprechende Fälle sind außerhalb der USA nicht bekannt. Derartige Beimischungen sind in Deutschland nicht handelsüblich.

Hinsichtlich der gesundheitlichen Risiken durch Passivinhalation weist das Deutsche Krebsforschungszentrum (2020) auf Feinstaubpartikel, Nikotin und weitere Substanzen (etwa Aromen, Nitrosamine, Acetformaldehyd oder Propylenglycol, die Augen-, Rachen- und Atemwegsreizungen verursachen können) in der Raumluft hin, die beim Gebrauch von E-Zigaretten entstehen und von Nichtkonsument_innen eingeatmet werden können: Die Belastung durch Passivinhalation ist vermutlich deutlich geringer als bei herkömmlichem Zigarettenrauch und naturgemäß in Räumen mit niedriger Ventilation erhöht. Eine gesundheitsschädigende Belastung durch Passivinhalation kann insbesondere für vulnerable Personengruppen, wie etwa Menschen mit beeinträchtigtem Gesundheitszustand, Schwangere und Kinder keinesfalls ausgeschlossen werden.

Die Deutsche Gesellschaft für Pneumologie und Beatmungsmedizin (DGP, 2020) schätzt die langfristigen Gesundheitsgefahren durch den Gebrauch von E-Zigaretten als derzeit ungeklärt ein und warnt ausdrücklich vor jegli- 
chem Gebrauch (einschließlich zur Tabakentwöhnung). Sie weist zudem im Rahmen einer ad-hoc-Stellungnahme auf besondere Gefahren für junge Konsument_innen von E-Zigaretten in der aktuellen COVID-19-Pandemie hin. In einer Online-Untersuchung der Universität Stanford in den USA zeigte sich bei einer großen Stichprobe von Jugendlichen und jungen Erwachsenen ( $\mathrm{N}=4351 ; 13-24$ Jahre alt), die E-Zigaretten gebrauchen, ein 5-fach erhöhtes Risiko, an COVID-19 zu erkranken (Gaiha et al., 2020).

\section{Trägt der Gebrauch von E-Zigaretten zu einer Renormalisierung des Rauchens bei und konterkariert womöglich bisherige Erfolge der Tabakprävention?}

Deutschland ist international hinsichtlich der Umsetzung von Maßnahmen der Tabakkontrolle wie Rauchverboten in Autos, Kneipen und Restaurants oder Werbeverboten vergleichsweise zurückhaltend; der Bevölkerungsanteil an Konsument innen nikotinhaltiger Produkte ist relativ hoch (Kotz et al., 2018). Dennoch verläuft der Rückgang des Neueinstieg des Tabakrauchens insbesondere unter Kindern und Jugendlichen seit Jahren kontinuierlich. Lag die Raucherquote bei den 12- bis 17-Jährigen im Jahr 2001 noch bei $28 \%$, so sank sie auf $15 \%$ in 2008 (bevor E-Zigaretten in Deutschland verfügbar wurden ${ }^{4}$ ) und weiter deutlich auf den historischen Tiefstand von 6,6\% in 2019 (Orth \& Merkel, 2020). Auch die Einstellungen gegenüber dem Rauchen haben sich, gerade unter jungen Menschen, über die Zeit sehr stark bis hin zu einer „Denormalisierung des Rauchens" bzw. einer "Normalisierung des Nichtrauchens“" (Robert Koch Institut, 2008) gewandelt. Kinder und Jugendliche haben von den präventiven Maßnahmen demnach besonders profitiert. $\mathrm{Zu}$ nennen sind die Schaffung rauchfreier Lebenswelten (z. B. in Schulen, Jugend- und Jugendhilfeeinrichtungen oder Vereinen), ein erschwerter Zugang zu Tabakwaren (etwa über Zigarettenautomaten und unzureichend kontrollierte Verkaufsstellen) sowie verhaltensbezogene präventive Maßnahmen (etwa im Rahmen von frühen und großflächig angelegten Klassenwettbewerben oder Lebenskompetenzprogramme an den allgemeinbildenden Schulen) (vgl. Arnaud \& Thomasius, 2020b).

Der weltweite Aufwärtstrend in der Nutzung von E-Zigaretten hingegen lässt die Befürchtung eines erheblichen bevölkerungsbezogenen Schadenspotenzials zu (DKFZ, 2015). Unter Jugendlichen in den USA (American Medical Association, 2019; Centers for Disease Control and Prevention, 2018) hat die Verfügbarkeit von nikotinhaltigen E-Zigaretten bereits zu einer neuen Epidemie der Nikotinabhängigkeit mit bislang völlig ungeklärten langfristigen gesundheit- lichen Auswirkungen (siehe vorhergehender Absatz zu den kurz-und langfristigen Risiken) geführt (ebd.). Angesichts der Popularität und dem Anstieg der jugendlichen Nutzerzahlen nikotinhaltiger E-Zigaretten ist auch in Deutschland eine mögliche „Renormalisierung“ des Rauchens zu befürchten, welche ähnlich den Entwicklungen in den USA gerade die unter Jugendlichen über Jahrzehnte errungenen Erfolge in der Prävention des Tabakkonsums gefährdet.

Eine solche Einschätzung ergibt sich zum einen aus den verfügbaren Daten zur Verbreitung des E-Zigarettenkonsums (siehe Absatz Wie verbreitet ist der E-Zigarettenkonsum im Kindes- und Jugendalter?); diese Daten lassen vermuten, dass neben den erwachsenen „Umsteigern“ von Tabak- auf E-Zigaretten insbesondere junge Menschen, die vormals noch nie Tabakzigaretten geraucht hatten und an sich ein eher niedrigeres diesbezügliches Risiko aufweisen (vgl. ESPAD, 2020; Hansen et al., 2020; Morgenstern et al., 2018; Schneider et al. 2016), überhaupt mit dem Rauchen zu beginnen, die höchsten Prävalenzen aufwiesen. (Für die Frage, ob der Konsum von E-Zigaretten das Risiko erhöht, später mit dem Konsum brennbarer Tabakzigaretten $\mathrm{zu}$ beginnen siehe Absatz Bahnt die Nutzung der E-Zigarette bei Jugendlichen den Übergang zum Tabakrauchen an?).

Zudem sind Befragungen zu den Einstellungen und Konsummotiven aufschlussreich. Eine Studie von Kotz und Kastaun (2018) berichtet abweichende Konsummotive Jugendlicher im Vergleich zu erwachsenen Nutzer_innen. Während die über 18-jährigen Befragten in einer großangelegten Repräsentativbefragung die E-Zigarette insbesondere als kostengünstigere und weniger gesundheitsschädliche Alternative zum Tabakrauchen sahen, waren die vier wesentlichen Gründe für eine Nutzung von E-Zigaretten bei den 14- bis 17-Jährigen 1. „weil es Spaß macht“, 2 . „weil es besser schmeckt als Tabak“, 3. „weil es cool/modern ist" und 4. „weil es viele Geschmacksrichtungen gibt".

Diese Ergebnisse zeigen, dass es einen erheblichen Unterschied gibt zwischen erwachsenen Tabakrauchern, die einen potenziellen gesundheitlichen und/oder ökonomischen Nutzen durch einen Umstieg von der Tabak- auf die E-Zigarette sehen und den meist jungen Zielgruppen, die direkt durch Werbung oder indirekt durch einen Wandel in der sozialen Wahrnehmung, Akzeptanz und „Normalisierung" in den peer-groups zu einem Einstieg in die E-Zigarettennutzung animiert werden.

Werbung für E-Zigaretten inszeniert das Rauchritual ähnlich der traditionellen Tabakwerbung als angenehm, entspannend, stilvoll oder romantisch (Jackler et al., 2019; Cantrell et al., 2017). Eine besonders für junge Menschen wichtige Rolle spielen die teils sehr aggressiven und mit großen Budgets ausgestatteten Vermarktungsstrategien der Produkte. Über multiple Kanäle verbreitete Werbebot-

In Deutschland liegen erst seit 2012 belastbare Daten zur Verbreitung der E-Zigarettennutzung vor. 
schaften entsprechen den oben genannten und von den Jugendlichen berichteten Konsummotiven. Die Art der Werbung für E-Zigaretten, etwa im Falle von JUUL über „,influencer" in sozialen Medien (Huang et al., 2019) spricht junge Menschen besonders an und führt bei Jugendlichen nachweislich auch zu mehr Konsum von E-Zigaretten (Auf et al., 2018; Hanewinkel et al., 2011; Hansen et al., 2018).

E-Zigaretten werden aktuell nicht nur als vermeintlich risikoarme Alternative zum Tabakrauchen vermarktet, sondern jungen Menschen sehr gezielt als trendiges Lifestyle-Produkt in attraktivem Design und fruchtigen und süßigkeitenähnlichen Aromen schmackhaft gemacht. Mit diesen Merkmalen weist die Vermarktung von E-Zigaretten sehr deutliche Parallelen zu den Ende der 1990er Jahre als Einstiegsprodukt in den Alkoholmarkt für junge Konsumenten konzipierten „Alkopops“ auf (DKFZ, 2014). Diese süß aromatisierten, spirituosenhaltigen Mischgetränke wurden bei jungen Menschen bis zur Einführung des Alkopopsteuergesetz im Jahr 2004 (mit einer deutlichen Teuerung) sehr stark nachgefragt und führten zu einer drastischen Steigerung des berichteten Alkoholkonsums (Müller et al., 2010). Auch die aktuelle Vermarktung gesüßter hochprozentiger Spirituosen in verschiedensten, den Alkoholgeschmack überdeckenden Geschmacksrichtungen (z.B. „Bubble Gum“, „Coco Bisquit”, „Luxury Lakritz”, „Magic Mango”) folgt demselben Muster: Genau wie EZigaretten werden sie in vielfältigen, fruchtigen und süßigkeitenähnlichen Geschmacksrichtungen angeboten und in bunter Aufmachung als cooles Lifestyle-Produkt für trendbewusste Jugendliche positioniert.

\section{Bahnt die Nutzung der E-Zigarette bei Jugendlichen den Übergang zum Tabakrauchen an?}

Für die Bewertung des Gefahrenpotenzials von E-Zigaretten sind nicht alleine die gesundheitlichen Risiken ausschlaggebend, sondern auch die Auswirkungen der Verfügbarkeit von E-Zigaretten auf die langfristige Entwicklung der Prävalenz des herkömmlichen Tabakkonsums (und zwar unabhängig davon, dass der Konsum des brennbaren Tabaks nach derzeitigem Stand des Wissens als gesundheitsschädigender eingeschätzt wird, siehe oben).

Von besonderer Bedeutung ist dabei die Frage, ob Jugendliche, die ansonsten nicht in den Tabakkonsum eingestiegen wären, durch den Konsum von E-Zigaretten dazu ermutigt werden (in der Fachliteratur wird diese Frage substanzübergreifend unter dem Begriff der „Gateway Hypothese" diskutiert; vgl. Lee \& Fry, 2019). Ob der Konsum von E-Zigaretten das Risiko von jungen Menschen für einen Einstieg bzw. eine Steigerung der Konsumintensität (etwa die Menge und Häufigkeit oder die Transition eines episodisch-experimentellen Gebrauchs in regelmäßige Konsummuster) erhöht, kann anhand von längsschnittlichen Studien eingeschätzt werden.

Ein Positionspapier der US-amerikanischen National Academy of Sciences (2018) erkennt auf der Grundlage der verfügbaren Studien deutliche Hinweise (,substantial evidence") dafür, dass durch einen E-Zigarettenkonsum das Risiko eines Jemalskonsums brennbarer Tabakprodukte bei Jugendlichen und jungen Erwachsenen steigt. Zudem werden moderate Hinweise (,moderate evidence“) für eine Steigerung des Tabakzigarettenkonsums bei tabakerfahrenen Konsument_innen durch E-Zigarettenkonsum konstatiert.

Grundlage dieser Einschätzung ist vor allem eine MetaAnalyse (Soneji et al., 2017) auf Basis von neun US-amerikanischen (längsschnittlichen) Kohortenstudien, in denen insgesamt 17389 Jugendliche und junge Erwachsene eingeschlossen wurden. Die Auswertung zeigt zusammenfassend und unter Berücksichtigung relevanter Kovariate, dass ein vormaliger Konsum von E-Zigaretten mit einer deutlichen Erhöhung des Risikos eines zeitlich nachgelagerten Jemalskonsum brennbarer Tabakzigaretten (Odds Ratio [OR] 3.62 [95\% CI, 2.42-5.41]) einherging. Die Wahrscheinlichkeit eines Tabakkonsums in den letzten 30 Tagen, die epidemiologisch als Hinweis auf einen regelmäßigen bzw. aktuellen Konsum gelten kann, war für vormalig E-Zigarettenerfahrene etwa um den Faktor 4 erhöht (OR 4.28 [95\% CI, 2.52-7.27]).

Ähnliche Ergebnisse zeigten sich in einer großangelegten US-amerikanischen prospektiven und repräsentativen Untersuchung mit über 6000 Jugendlichen (12 bis 15 Jahre alt) ohne vorherigen Tabakkonsum (Berry et al., 2019). Hier war bei den Jemalskonsument_innen von E-Zigaretten sowohl das Risiko eines prospektiven Jemals- als auch eines Aktualkonsums von Tabak innerhalb von zwei Jahren ebenfalls um ein 4-faches (OR 4.09; 95\% CI, 2.97-5.63; Jemalskonsum) bzw. fast ein 3-faches (OR 2.75; 95\% CI, 1.60-4.73; Aktualkonsum) erhöht. Interessanterweise war das Einstiegsrisiko in den Tabakkonsum bei jugendlichen E-Zigarettenkonsument_innen mit einer ansonsten vergleichsweise niedrigen Tendenz für risikoreiche Verhaltensweisen (einschließlich des Konsums weiterer psychoaktiver Substanzen) mit einem über 8-fachen Risiko besonders stark ausgeprägt (OR, 8.57; 95\% CI, 3.87-18.97).

Daten aus europäischen Ländern sind nicht in vergleichbarer Fülle und Qualität vorhanden, weisen vom Ergebnis her aber in eine ähnlich eindeutige Richtung. In einer prospektiven britischen Schüleruntersuchung (Conner et al., 2018; $N=2836 ; 20$ Schulen in England, 13 bis 14 Jahre, Beobachtungszeitraum 12 Monate) zeigte sich ebenfalls ein deutlicher und für bekannte Risikofaktoren für den Einstieg ins Tabakrauchen statistisch kontrollierter $\mathrm{Zu}-$ sammenhang zwischen dem Konsum von E-Zigaretten und der nachfolgenden Initiation des Tabakrauchens; die 
Risikoerhöhung betrug wiederum etwa das 4 -fache (OR 4.06, 95\% CI 2.94-5.60).

Aus Deutschland liegen ebenfalls relevante Studien vor, welche die internationalen Befunde insgesamt bestätigen. In einer aktuellen Untersuchung (Hansen et al., 2020) wurden 2388 Schüler_innen (mittleres Alter: 11,8 Jahre, 49,6\% weiblich) aus mehreren Bundesländern (Baden-Württemberg, Mecklenburg-Vorpommern, Nordrhein-Westfalen, Rheinland-Pfalz, Schleswig-Holstein und Sachsen), die nie zuvor konventionelle Zigaretten geraucht hatten, über zwei Jahre zu ihrem Konsumverhalten befragt. Die Auswertung ergab eine Steigerung des relativen Risikos für das Experimentieren mit konventionellen Zigaretten bei Nutzer_innen von E-Zigaretten um $85 \%$ (adjustiertes relatives Risiko $=1,85 ; 95 \%$ CI 1.34-2.56). Wie in den oben berichteten internationalen Studien wurden bekannte alternative Risikofaktoren (Alter, Geschlecht, Migrationshintergrund, „Sensation Seeking“, Schulleistung, Alkoholkonsum, sozioökonomischem Status und Schulart) in den Analysen statistisch kontrolliert. Auch in dieser Studie zeigte sich eine stärkere Assoziation zwischen vormaligem E-Zigarettenkonsum und einer Neigung zum Experimentalkonsum von Tabakzigaretten bei Kindern und Jugendlichen, die ein generell niedrigeres Risiko haben, mit dem Rauchen zu beginnen (charakterisiert etwa durch niedriges „Sensation Seeking“).

Diese Befunde bestätigen eine ähnlich konzipierte frühere Studie aus derselben Arbeitsgruppe (Morgenstern et al., 2018) bei 2186 etwas älteren Jugendlichen (Zehntklässlern) aus Niedersachsen und Schleswig-Holstein (mittleres Alter: 15,5 Jahre, 53,6\% weiblich), die ebenfalls niemals zuvor konventionelle Zigaretten geraucht hatten. Die Studie kommt im Kern zu identischen Ergebnissen: Das relative Risiko für das Experimentieren mit Tabak war im 6-monatigen Beobachtungszeitraum (wiederum kontrolliert für bekannte Risikofaktoren) bei Nutzern von EZigaretten um das 2,2-fache erhöht (adjustiertes relatives Risiko $=2,18$; $95 \%$ CI 1,65-2,83) und besonders ausgeprägt für Jugendliche mit niedrigem „Sensation Seeking“ und ohne Alkoholrausch-Erfahrung.

Zusammengefasst lässt sich sagen, dass sich auf Basis methodisch hochwertiger (längschnittlicher) internationaler und in Deutschland durchgeführter Studien ein klares Bild erkennen lässt: der Konsum von E-Zigaretten erhöht („,bahnt“) die Wahrscheinlichkeit eines Einstiegs in den Konsum konventioneller Tabakzigaretten bei jungen Menschen ohne vorherige Konsumerfahrung erheblich. Der Zusammenhang scheint stärker zu sein für Jugendliche, die generell ein niedrigeres Risiko haben, mit dem Rauchen zu beginnen. Zukünftige Studien sollten daher einerseits die spezifischen Risikofaktoren des Einstiegs in den E-Zigarettenkonsums ermitteln, als auch diejenigen der Transition eines episodisch-experimentellen Ge- brauchs in regelmäßige und abhängige Tabakkonsummuster (vgl. Schneider \& Diehl, 2016).

\section{Zusammenfassung und Schlussfolgerungen}

Die in dem vorliegenden Beitrag zusammengefassten Befunde ergeben ein klares Bild. E-Zigaretten sind für Kinder und Jugendliche besonders gefährlich. Mit der E-Zigarette werden nach Auffassung der deutschen Ärzte für Kinderund Jugendpsychiatrie und Psychotherapie die großen Erfolge der Tabakprävention der letzten Jahre unterlaufen und in ihr Gegenteil verkehrt.

Es gibt weiter einen Rückgang des Tabakkonsums bei Kindern und Jugendlichen zu verzeichnen, der in deutlichem Kontrast zur Zunahme des Konsums von E-Zigaretten bei Jugendlichen steht. Um das Risiko dieser Entwicklung zu verstehen, müssen die Besonderheiten der jungen Altersgruppe berücksichtigt werden: Während E-Zigaretten manche Erwachsene gegebenenfalls dabei unterstützen können, zeitweise auf Tabakprodukte zu verzichten, erhöhen sie für Kinder und Jugendliche sowohl das Risiko der Entwicklung einer Nikotinabhängigkeit als auch das Einstiegsrisiko für das Rauchen von Tabakzigaretten. Junge Nie-Raucher konsumieren häufiger E-Zigaretten als Gleichaltrige mit einem gelegentlichen und regelmäßigen Tabakgebrauch (ESPAD, 2020) und experimentieren häufiger mit konventionellen Zigaretten, wenn sie zuvor E-Zigaretten konsumiert haben. Dieser Einfluss fällt aktuellen Studien zufolge (ESPAD, 2020; Hansen et al., 2020) vor allem in der Gruppe derjenigen Jugendlichen auf, die an sich ein generell niedrigeres Risiko haben, überhaupt mit dem Rauchen zu beginnen.

Die Art der Werbung für E-Zigaretten spricht junge Menschen besonders an. Jugendliche werden durch die Aufmachung der Werbung, "trendige“ Produkte mit puristischem Design und den aromatischen Geschmack gezielt zum Konsum animiert. In diesen Merkmalen gibt es sehr deutliche Parallelen mit entsprechenden Produkten der Alkoholindustrie (DKFZ, 2014). Werbung für E-Zigaretten führt bei Jugendlichen nachweislich auch zu mehr Konsum von E-Zigaretten (Auf et al., 2018; Hanewinkel et al., 2011; Hansen et al., 2018). E-Zigaretten verharmlosen Gefahren des Nikotinkonsums, indem sie Warnsignale herkömmlicher Zigaretten (bitterer Geschmack, Rauch) überstrahlen oder unterdrücken. Die habituelle Verknüpfung vermeintlich „cleaner“ Produkte mit modernem Lifestyle und Werten wie Gesundheit, Gemeinschaftserleben und Leistungsfähigkeit muss als besonders gefährlich angesehen werden. Für Jugendliche sind 
E-Zigaretten aber eben nicht harmlose Lifestyle-Produkte und auch nicht die „beste Alternative zur Zigarette“. Die von den Herstellern propagierte Botschaft von der "rauchfreien Zukunft", gekoppelt mit neuen Produkten und intensiver Werbung, gefährdet die Erfolge der Tabakprävention der letzten Jahre. Innovative Prävention muss über dieses Zerrbild des "gesunden Rauchens" aufklären. Das von der Bundesregierung geplante Tabakwerbeverbot muss zum Schutz von Kindern und Jugendlichen daher auch für E-Zigaretten gelten.

In den USA hat die Zahl Minderjähriger, die E-Zigaretten nutzen, bereits dramatisch zugenommen. Die Suchtkommission der deutschen kinder- und jugendpsychiatrischen Verbände und wissenschaftlichen Fachgesellschaft spricht sich für ein umgehendes, striktes und umfassendes Werbeverbot für E-Zigaretten und für alle Tabakprodukte aus und plädiert für wirksame Maßnahmen zur Durchsetzung des Jugendschutzgesetzes sowie für eine unabhängige Erforschung der Risiken der E-Zigaretten.

\section{Literatur}

Arnaud, N. \& Thomasius, R. (2020b). Prävention von stoffgebundenen Suchtstörungen. Zeitschrift für Kinder- und Jugendpsychiatrie und Psychotherapie, 48, 381-392. https://doi.org/10.10 24/1422-4917/a000636

Arnaud, N. \& Thomasius, R. (2020a). Störungen durch Substanzgebrauch und abhängige Verhaltensweisen in der ICD-11. Zeitschrift für Kinder- und Jugendpsychiatrie und Psychotherapie [Vorab-Onlinepublikation], 1-8. https://doi.org/10.1024/14224917/a000748

American Medical Association. (2019). E-cigarettes and vaping: A public health epidemic. Zugriff am 19. August 2020 unter https://www.ama-assn.org/delivering-care/public-health/ e-cigarettes-and-vaping-public-health-epidemic

Auer, R., Concha-Lozano, N., Jacot-Sadowski, I., Cornuz, J. \& Berthet, A. (2017). Heat-not-burn tobacco cigarettes: Smoke by any other name. JAMA Internal Medicine, 177, 1050-1052.

Auf, R., Trepka, M.J., Selim, M., Taleb, Z.B., De La Rosa, M. \& Cano, M.Á. (2018). E-cigarette marketing exposure and combustible tobacco use among adolescents in the United States. Addictive Behaviors, 78, 74-79.

Benowitz, N.L. \& Burbank, A.D. (2016). Cardiovascular toxicity of nicotine: Implications for electronic cigarette use. Trends in Cardiovascular Medicine, 26, 515-523.

Berry, K. M., Fetterman, J.L., Benjamin, E.J., Bhatnagar, A., Barrington-Trimis, J. L., Leventhal, A. M. \& Stokes, A. (2019). Association of electronic cigarette use with subsequent initiation of tobacco cigarettes in US youths. JAMA Network Open, 2, e187794-e187794.

Bhatta, D. N. \& Glantz, S.A. (2020). Association of e-cigarette use with respiratory disease among adults: A longitudinal analysis. American Journal of Preventive Medicine, 58, 182-190.

Blount, B.C., Karwowski, M.P., Shields, P.G., Morel-Espinosa, M., Valentin-Blasini, L., Gardner, M., Braselton, M., Brosius, C.R., Caron, K.T., Chambers, D., Corstvet, J., Cowan, E., De Jesús, V.R., Espinosa, P., Fernandez, C., Holder, C., Kuklenyik, Z., Kusovschi, J.D., Newman, C., ... Pirkle, J.L. (2020). Vitamin E Acetate in Bronchoalveolar-Lavage Fluid Associated with EVALI. New England Journal of Medicine, 382, 697-705.
Cantrell, J., Ganz, O., Emelle, B., Moore, R., Rath, J., Hair, E.C. \& Vallone, D. (2017). Mobile marketing: An emerging strategy to promote electronic nicotine delivery systems. Tobacco Control, 26, e1-e3.

Centers for Disease Control and Prevention. (2018). Surgeon General's Advisory on E-cigarette Use Among Youth. Zugriff am 01 Dezember 2020 unter https://e-cigarettes.surgeongeneral. gov/documents/surgeon-generals-advisory-on-e-cigaretteuse-among-youth-2018.pdf

Conner, M., Grogan, S., Simms-Ellis, R., Flett, K., Sykes-Muskett, B., Cowap, L., Lawton, R., Armitage, C.J., Meads, D. \& Torgerson, C. (2018). Do electronic cigarettes increase cigarette smoking in UK adolescents? Evidence from a 12-month prospective study. Tobacco Control, 27, 365-372.

Cullen, K.A., Gentzke, A.S., Sawdey, M.D., Chang, J.T., Anic, G.M., Wang, T.W., Creamer, M. R., Jamal, A., Ambrose, B. K. \& King, B. A. (2019). E-Cigarette Use Among Youth in the United States, 2019. JAMA, 322, 2095-2103.

Deutsche Hauptstelle für Suchtfragen e.V. (DHS). (2016). „Harm Reduction": Verringerung von tabakrauchbedingten Gesundheitsschäden durch E-Zigaretten? Stellungnahme der Deutschen Hauptstelle für Suchtfragen e.V. Zugriff am 15. August 2020 unter https://www.dhs.de/fileadmin/user_upload/pdf/ dhs_stellungnahmen/DHS_Positionspapier_Harm_Reduction. pdf.

Deutsches Krebsforschungszentrum (DKFZ) e.V. (2020). Factsheet prepared for the 8th European Conference on Tobacco or Health, 19 to 22 February 2020, Berlin, Germany. Zugriff am 15. August 2020 unter https://www.dkfz.de/de/tabakkontrolle/download/ Publikationen/sonstVeroeffentlichungen/ECToH-2020_Factsheets_all.pdf? $m=1582544222 \&$

Deutsches Krebsforschungszentrum (DKFZ) e.V. (2015). Memorandum zur gesetzlichen Regulierung von nikotinhaltigen und nikotinfreien E-Zigaretten. Zugriff

am 19. September 2020 unter http://www.mpr-online.de.https:// www.dkfz.de/de/tabakkontrolle/download/Publikationen/Me morandum/Memorandum_2015_E-Zigarette.pdf

Deutsches Krebsforschungszentrum. (2014). Marketing für E-Zigaretten in Deutschland. Zugriff am 19. September 2020 unter https://www.dkfz.de/de/tabakkontrolle/download/Publikationen/ RoteReihe/Band_20_Marketing_fuer_E-Zigaretten_in_ Deutschland.pdf

Eaton, D. L., Kwan, L.Y. \& Stratton, K. (Eds.). (2018). Public Health Consequences of E-Cigarettes. Washington (DC): National Academies Press. Zugriff am 19. Juli 2020 unter https://www.ncbi. nlm.nih.gov/books/NBK507163/

Eichler, M., Blettner, M. \& Singer, S. (2016). The use of e-cigarettes: A population-based cross-sectional survey of 4002 individuals in 2016. Deutsches Ärzteblatt International, 113, 847.

Ernst \& Young. (2017). Electronic Nicotine Delivery Systems (ENDS): An Update on a Rapidly Evolving Vapour Market Report 2, 1-25.

ESPAD Group. (2020). ESPAD Report 2019: Results from the European School Survey Project on Alcohol and Other Drugs. Luxembourg: Publications Office of the European Union. Zugriff am 19. November 2020 unter http://www.espad.org/sites/espad. org/files/2020.3878_EN_04.pdf

Gaiha, S.M., Cheng, J. \& Halpern-Felsher, B. (2020). Association Between Youth Smoking, Electronic Cigarette Use, and COVID-19. Journal of Adolescent Health, 67, 519-523.

George, J., Hussain, M., Vadiveloo, T., Ireland, S., Hopkinson, P., Struthers, A. D., Donnan, P.T., Khan, F. \& Lang, C.C. (2019). Cardiovascular Effects of Switching From Tobacco Cigarettes to Electronic Cigarettes. Journal of the American College of Cardiology, 74, 3112-3120.

Gotts, J.E., Jordt, S.-E., McConnell, R. \& Tarran, R. (2019). What are the respiratory effects of e-cigarettes? British Medical Journal, 366,15275 . 
Hanewinkel, R., Isensee, B., Sargent, J. D. \& Morgenstern, M. (2011). Cigarette advertising and teen smoking initiation. Pediatrics, 127, e271-e278.

Hansen, J., Hanewinkel, R. \& Morgenstern, M. (2018). Electronic cigarette marketing and smoking behaviour in adolescence: A cross-sectional study. ERJ Open Research, 4, 00155-02018. https://doi.org/10.1183/23120541.00155-2018

Hansen, J., Janssen, J., Morgenstern, M. \& Hanewinkel, R. (2020). E-Zigarettenkonsum und späterer Konsum konventioneller Zigaretten: Ergebnisse einer 2-jährigen prospektiven Beobachtungsstudie. Pneumologie, 74, 39-45.

Hartmann-Boyce, J., McRobbie, H., Lindson, N., Bullen, C, Begh, R., Theodoulou, A., Notley, C., Rigotti, N.A., Turner, T., Butler, A.R. \& Hajek, P. (2020). Electronic cigarettes for smoking cessation. Cochrane Database of Systematic Reviews, 10, CD010216. https://doi.org/10.1002/14651858.CD010216.pub4

Huang, J., Duan, Z., Kwok, J., Binns, S., Vera, L. E., Kim, Y., Szczypka, G. \& Emery, S. L. (2019). Vaping versus JUULing: How the extraordinary growth and marketing of JUUL transformed the US retail e-cigarette market. Tobacco control, 28, 146-151.

Jackler, R. K., Chau, C., Getachew, B., Whitcomb, M., Lee-Heidenreich, J., Bhatt, A. \& Ramamurthi, D. (2019). JUUL advertising over its first three years on the market. SRITA White Paper. Zugriff am 16. August 2020 unter http://tobacco.stanford.edu/ tobacco_main/publications/JUUL_Marketing_Stanford.pdf

Jankowski, M., Krzystanek, M., Zejda, J.E., Majek, P., Lubanski, J., Lawson, J.A. \& Brozek, G. (2019). E-cigarettes are more addictive than traditional cigarettes - A study in highly educated young people. International Journal of Environmental Research and Public Health, 16, 2279.

Johnson, J.M., Muilenburg, J.L., Rathbun, S.L., Yu, X., Naeher, L.P. \& Wang, J.-S. (2018). Elevated nicotine dependence scores among electronic cigarette users at an electronic cigarette convention. Journal of Community Health, 43, 164-174.

Kandel, E.R. \& Kandel, D.B. (2014). A molecular basis for nicotine as a gateway drug. New England Journal of Medicine, 371, 932-943.

Kotz, D., Böckmann, M. \& Kastaun, S. (2018). The Use of Tobacco, ECigarettes, and Methods to Quit Smoking in Germany: A Representative Study Using 6 Waves of Data Over 12 Months (the DEBRA Study). Deutsches Ärzteblatt International, 115, 235-242.

Kotz, D. \& Kastaun, S. (2018). E-Zigaretten und Tabakerhitzer: Repräsentative Daten zu Konsumverhalten und assoziierten Faktoren in der deutschen Bevölkerung (die DEBRA-Studie). Bundesgesundheitsblatt-Gesundheitsforschung-Gesundheitsschutz, 61,1407-1414.

Lee, P. \& Fry, J. (2019). Investigating gateway effects using the PATH study. F1000Research, 8, 264. https://doi.org/10.12688/f1000 research.18354.2

Lisko, J.G., Tran, H., Stanfill, S.B., Blount, B.C. \& Watson, C.H. (2015). Chemical composition and evaluation of nicotine, tobacco alkaloids, $\mathrm{pH}$, and selected flavors in e-cigarette cartridges and refill solutions. Nicotine \& Tobacco Research, 17, 1270-1278.

Mishra, V. K., Kim, K.-H., Samaddar, P., Kumar, S., Aggarwal, M., Chacko, K., Mishra, V.K., Kim, K.-H., Samaddar, P. \& Kumar, S. (2017). Review on metallic components released due to the use of electronic cigarettes. Environmental Engineering Research, 22, 131-140.

Morgenstern, M., Nies, A., Goecke, M. \& Hanewinkel, R. (2018). EZigaretten und der Einstieg in den Konsum konventioneller Zigaretten. Deutsches Ärzteblatt, 115, 243-248.

Müller, S., Piontek, D., Pabst, A., Baumeister, S. E. \& Kraus, L. (2010). Changes in alcohol consumption and beverage preference among adolescents after the introduction of the alcopops tax in Germany. Addiction, 105, 1205-1213.
National Centre for Smoking Cessation and Training (NCSCT). (2016). Electronic cigarettes: A briefing for stop smoking services. https://www.ncsct.co.uk/usr/pub/Electronic_cigarettes._A_briefing_for_stop_smoking_services.pdf; letzter Zugriff: 15.10.2020

Nicol, J., Fraser, R., Walker, L., Liu, C., Murphy, J. \& Proctor, C.J. (2020). Comprehensive Chemical Characterization of the Aerosol Emissions of a Vaping Product Based on a New Technology. Chemical Research in Toxicology, 33, 789-799.

Nowak, D., Gohlke, H., Hering, T., Herth, F., Jany, B., Raupach, T., Welte, T. \& Loddenkemper, R. (2015). Positionspapier der Deutschen Gesellschaft für Pneumologie und Beatmungsmedizin eV (DGP) zur elektronischen Zigarette (E-Zigarette). Pneumologie, 69, 131-134.

Orth, B. \& Merkel, C. (2018). The decline of cigarette smoking among adolescents and young adults in Germany and the rising relevance of waterpipes, e-cigarettes and e-hookahs. Bundesgesundheitsblatt, Gesundheitsforschung, Gesundheitsschutz, 61, 1377.

Orth, B. \& Merkel, C. (2020). Die Drogenaffinität Jugendlicher in der Bundesrepublik Deutschland 2019. Rauchen, Alkoholkonsum und Konsum illegaler Drogen: Aktuelle Verbreitung und Trends. BZgA-Forschungsbericht. Köln: Bundeszentrale für gesundheitliche Aufklärung. Zugriff am 7. August 2020 unter https://doi. org/10.17623/BZGA:225-DAS19-DE-1.0

Pisinger, C. \& Døssing, M. (2014). A systematic review of health effects of electronic cigarettes. Preventive Medicine, 69, 248-260.

Reimer, J., Kuhn, S. \& Lehmann, K. (2016). Konsumgewohnheiten und Motive von E-Zigaretten-Konsumenten in DeutschlandEine Querschnittsanalyse. Zugriff am 7. August 2020 unter https://www.bundesgesundheitsministerium.de/fileadmin/ Dateien/5_Publikationen/Drogen_und_Sucht/Berichte/16100 5_Anlage_5-Abschlussbericht_ZIS.pdf

Robert Koch Institut. (2008). Erkennen-Bewerten-Handeln: Zur Gesundheit von Kindern und Jugendlichen in Deutschland. Zugriff am 4. August 2020 unter https://www.rki.de/DE/Content/ Gesundheitsmonitoring/Studien/Kiggs/Basiserhebung/KiGG S_GPA.pdf?__blob=publicationFile

Rüther, T., Backmund, M., Bischof, G., Lange, N., Missel, P., Preuß, U., Rumpf, H.-J., Thomasius, R. \& Batra, A. (2017). Positionspapier: Suchtmedizinische und gesundheitspolitische Chancen und Risiken durch den Gebrauch von E-Zigaretten. Suchttherapie, 18, 120-123.

Schneider, S. \& Diehl, K. (2016). Vaping as a catalyst for smoking? An initial model on the initiation of electronic cigarette use and the transition to tobacco smoking among adolescents. Nicotine \& Tobacco Research, 18, 647-653.

Schneider, S., Görig, T., Schilling, L. \& Diehl, K. (2017). E-Zigaretten in aller Munde?-Aktuelle repräsentative Daten zur Nutzung unter Jugendlichen und Erwachsenen. DMW-Deutsche Medizinische Wochenschrift, 142, e156-e166.

Soneji, S., Barrington-Trimis, J.L., Wills, T.A., Leventhal, A. M., Unger, J.B., Gibson, L.A., Yang, J., Primack, B.A., Andrews, J.A. \& Miech, R.A. (2017). Association between initial use of e-cigarettes and subsequent cigarette smoking among adolescents and young adults: A systematic review and meta-analysis. JAMA Pediatrics, 171, 788-797.

Suchtkommission der deutschen kinder-, jugendpsychiatrischen Verbände und wissenschaftlichen Fachgesellschaft (BAG KJPP, BKJPP, DGKJP). (2020). E-Zigaretten erhöhen Einstiegsrisiko bei Jugendlichen für Zigaretten-Rauchen: Umfassendes Werbeverbot für E-Zigaretten und für alle Tabakprodukte notwendig. Zeitschrift für Kinder- und Jugendpsychiatrie und Psychotherapie, 48, 87-88.

Wang, T.W., Neff, L.J., Park-Lee, E., Ren, C., Cullen, K.A. \& King, B.A. (2020). E-cigarette Use Among Middle and High School Students - United States. Morbidity Mortality Weekly Report, 69, 1310-1312. https://doi.org/10.15585/mmwr.mm6937e1 
Yoong, S.L., Stockings, E., Chai, L. K., Tzelepis, F., Wiggers, J., Oldmeadow, C., Paul, C., Peruga, A., Kingsland, M. \& Attia, J. (2018). Prevalence of electronic nicotine delivery systems (ENDS) use among youth globally: A systematic review and meta-analysis of country level data. Australian and New Zealand Journal of Public Health, 42, 303-308.

\section{Historie}

Manuskript eingereicht: 10.12.2020

Nach Revision angenommen: 29.07.2021

Onlineveröffentlichung: 14.10.2021

\section{Anmerkung}

Der Absatz „Zusammenfassung und Schlussfolgerungen“ ist bereits als Kurzversion der vorliegenden Stellungnahme vorab publiziert worden (Suchtkommission der deutschen kinder-, jugendpsychiatrischen Verbände und wissenschaftlichen Fachgesellschaft (BAG KJPP, BKJPP, DGKJP, 2020).

\section{Förderung}

Open-Access-Veröffentlichung ermöglicht durch

die Universität Hamburg.

Interessenskonflikt

Es bestehen keine Interessenskonflikte.

Dr. phil. Dipl.-Psych. Nicolas Arnaud

Deutsches Zentrum für Suchtfragen des Kindesund Jugendalters (DZSKJ)

Universitätsklinikum Hamburg-Eppendorf

Martinistr. 52

20246 Hamburg

Deutschland

n.arnaud@uke.de

\section{CME-Fragen}

1. Frage: Welche Aussage ist falsch? (Einfachauswahl)

a. Die Entwicklung der Prävalenzraten des E-Zigarettenkonsums unter Jugendlichen ist unter anderem abhängig von der Verfügbarkeit und der gesetzgeberischen Regulation.

b. Internationale Daten legen nahe, dass Jugendliche mehrheitlich E-Zigarettenprodukte mit hohem Nikotingehalt konsumieren.

c. Jugendliche und Erwachsene unterscheiden sich in ihren Motiven für den Konsum von E-Zigaretten.

d. Der Anteil regelmäßiger E-Zigarettenkonsumenten ist unter den Jugendlichen in Deutschland höher als der Anteil regelmäßiger Konsumenten von Tabakprodukten.

\section{Frage: Auf Basis der derzeitigen Studienlage kön-} nen folgende Aussagen zu den gesundheitlichen Risiken des Konsums von E-Zigaretten gemacht werden (Einfachauswahl)

a. Regelmäßiger E-Zigarettenkonsum erhöht das Herzinfarktrisiko um den Faktor 2,25.

b. Die langfristigen Auswirkungen des Konsums von E-Zigaretten können in ihrer Vergleichbarkeit zu den Risiken des Konsums brennbarer Zigaretten derzeit nicht eingeschätzt werden.

c. Für das Abhängigkeitspotenzial von E-Zigaretten ist der Nikotingehalt nicht entscheidend.

d. Die gesundheitlichen Risiken des E-Zigarettenkonsums sind mit den Risiken des Konsums konventioneller Tabakzigaretten vergleichbar.
3. Frage: Im Zusammenhang mit dem Konsum von E-Zigaretten kam es 2019 zu einer Welle schwerer Lungenerkranken mit Todesfolge... (Einfachauswahl)

a. vor allem bei erwachsenen Konsument_innen in den USA.

b. vor allem bei Konsument_innen in Europa.

c. vermutlich durch die Beimischung THC-haltiger Liquids.

d. die bisher nicht aufgeklärt werden konnten.

\section{Frage: Aus den vorhandenen Studien zur Nutzung} von E-Zigaretten lässt sich ableiten, dass...

(Einfachauswahl)

a. Jugendliche, die mit dem E-Zigarettenkonsum beginnen, ein ähnliches Risikoprofil aufweisen wie diejenigen, die mit dem Konsum klassischer Tabakzigaretten beginnen.

b. Jugendliche, die über den Konsum von E-Zigaretten in den Tabakkonsum einsteigen, eine generelle Affinität für riskante Verhaltensweisen aufweisen.

c. Jugendliche, die E-Zigaretten konsumieren, in der Regel bereits vorher mit dem Konsum von Tabakzigaretten experimentiert haben.

d. Jugendliche, die Tabakzigaretten konsumieren, häufig bereits vorher mit dem Konsum von E-Zigaretten experimentiert haben.

5. Frage: Welches Konsummotiv ist unter jugendlichen Konsument_innen von E-Zigarette am wenigsten

üblich? (Einfachauswahl)

a. Weil es Spaß macht

b. Weil es besser schmeckt als Tabak

c. Weil es cool/modern ist

d. Weil es weniger gesundheitsschädlich ist als Tabakrauchen 
Um Ihr CME-Zertifikat zu erhalten (min. drei richtige Antworten), schicken Sie bitte den ausgefüllten Fragebogen mit einem frankierten Rückumschlag bis zum 30.04.2022 an die nebenstehende Adresse. Später eintreffende Antworten und solche ohne bzw. mit nicht frankierten Rückumschlägen können nicht mehr berücksichtigt werden.

\section{Milena Becker}

LWL-Universitätsklinik Hamm der Ruhr-Universität Bochum Klinik für Kinder- und Jugendpsychiatrie, Psychotherapie und Psychosomatik

Heithofer Allee 64

59071 Hamm, Deutschland

\section{Fortbildungszertifikat}

Die Ärztekammer Niedersachsen erkennt hiermit

2 Fortbildungspunkte an.

\section{Stempel \\ Kinder- und Jugend- psychiatrie und Psychotherapie $02 / 2022$ \\ (v) hogrefe}

Wichtig: Bitte kleben Sie hier Ihr Barcode-

Etikett (EFN) an oder schreiben Sie Ihre EFN.

Bitte teilen Sie uns alternativ Ihre

Psychotherapeuten-Fortbildungsnummer mit:
„E-Zigaretten bei Kindern und Jugendlichen“

Die Antworten bitte deutlich ankreuzen!

a

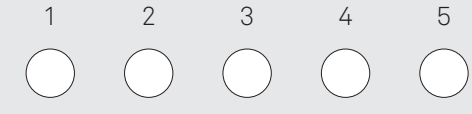

b
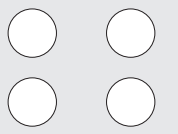

0

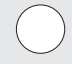

0
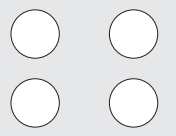

e
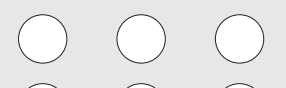

0
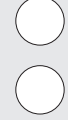

Ich versichere, alle Fragen ohne fremde Hilfe beantwortet zu haben.

Name

Berufsbezeichnung, Titel

Straße, Nr.

PLZ, Ort 